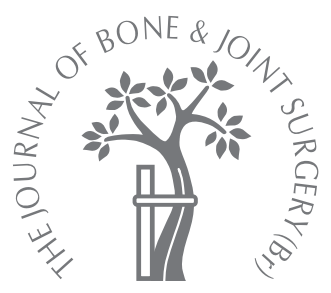

\title{
Total knee replacement with rotational proximal tibial osteotomy for osteoarthritis with severe external tibial torsion and patellar instability
}

R. Ramaswamy, Y. Kosashvili, H. U. Cameron, J. C. Cameron

From Holland Orthopaedic and Arthritic Centre, Toronto, Canada

n. Ramaswamy, MSc, FRCS(Trauma \& Orth), Clinical fellow in Lower Limb Joint Reconstruction

- Y. Kosashvili, MD, MHA, Clinical Fellow in Lower Limb Joint Reconstruction

H. U. Cameron, MD, FRCS C,

Consultant Orthopaedic

Surgeon

I. C. Cameron, MD, FRCS C,

Consultant Orthopaedic

Surgeon

Holland Orthopaedic and

Arthritic Centre, 43 Wellesley

Street East, Toronto, Ontario,

Canada, M4Y $1 \mathrm{H} 1$.

Correspondence should be sent to Dr Y. Kosashvili; e-mail: yonasofi@gmail.com

(C)2009 British Editorial Society of Bone and Joint Surgery doi:10.1302/0301-620X.91B11. $22658 \$ 2.00$

$J$ Bone Joint Surg [Br] 2009;91-B:1466-71.

Received 31 March 2009;

Accepted 29 June 2009

\author{
The management of osteoarthritis of the knee associated with patellar instability secondary \\ to external tibial torsion $>\mathbf{4 5}^{\circ}$ is challenging. Patellofemoral biomechanics in these patients \\ cannot be achieved by intra-articular correction using standard techniques of total knee \\ replacement. \\ We reviewed seven patients (eight knees) with recurrent patellar dislocation and one with \\ bilateral irreducible lateral dislocation who had undergone simultaneous total knee \\ replacement and internal tibial derotational osteotomy. All had osteoarthritis and severe \\ external tibial torsion. The mean follow-up was for $\mathbf{4 7 . 2}$ months (24 to 120). \\ The mean objective and functional Knee Society scores improved significantly \\ ( $p=0.0001$ ) from 29.7 and 41.5 pre-operatively to 71.4 and 73.5 post-operatively, \\ respectively. In all patients the osteotomies healed and patellar stability was restored. \\ Excessive external tibial torsion should be identified and corrected in patients with \\ osteoarthritis and patellar instability. Simultaneous internal rotation osteotomy of the tibia \\ and total knee replacement is a technically demanding but effective treatment for such \\ patients.
}

The various causes of instability of the patellofemoral joint include valgus alignment of the limb, trochlear hypoplasia, patella alta and external tibial torsion with a high $\mathrm{Q}$ angle. ${ }^{1,2}$ Of these, isolated excessive external tibial torsion is a rare, but under-recognised cause. . $^{1,3}$

The aetiology of external tibial torsion is multifactorial with genetics, the intrauterine position and mechanical forces all playing a role. The condition persists in the growing child with a level of torsion of $15^{\circ}$ to $20^{\circ}$ between the ages of 5 and 7 years. ${ }^{3-5}$ The diagnosis of excessive external torsion is based mainly on physical examination and should be distinguished from femoral anteversion. ${ }^{1}$ In order to do this the lower limb should be examined as a whole. With the patient supine, internal and external rotation of the hip is measured in $90^{\circ}$ of flexion. If this is symmetrical, excessive femoral anteversion is not the culprit. Rotation of the hips is then assessed with the hips in neutral and the feet perpendicular to the ground. If external rotation is significantly larger than internal rotation, and there are no extra-articular deformities such as malunion of a fracture, the underlying problem is external tibial torsion. The patient is examined prone, with the foot plantigrade, in order to determine the thigh-foot angle and to record the degree of external tibial torsion. The $Q$ angle is increased in patients with excessive external tibial torsion. However, it cannot be measured reliably when it is associated with chronic irreducible patellar dislocation. In such patients CT may provide additional data regarding the condition of the trochlea by measuring the tibial tuberosity-trochlear groove distance. $^{6,7}$ Although CT is the method of choice for quantifying tibial torsion, clinical examination generally yields information which is just as accurate. ${ }^{7}$

Patients with patellar instability secondary to external tibial torsion and osteoarthritis often present with an apparent valgus deformity as they try to improve their foot progression angle by internal rotation and adduction of their hips. Consequently, the knee is driven medially relative to the foot, thereby increasing the load through the lateral and patellofemoral compartments. Total knee replacement (TKR) for osteoarthritis associated with patellar instability secondary to excessive external tibial torsion is challenging. No more than $20^{\circ}$ of excessive external tibial torsion can be corrected reliably without a rotational tibial osteotomy, as the attempted derotation increases the tension 


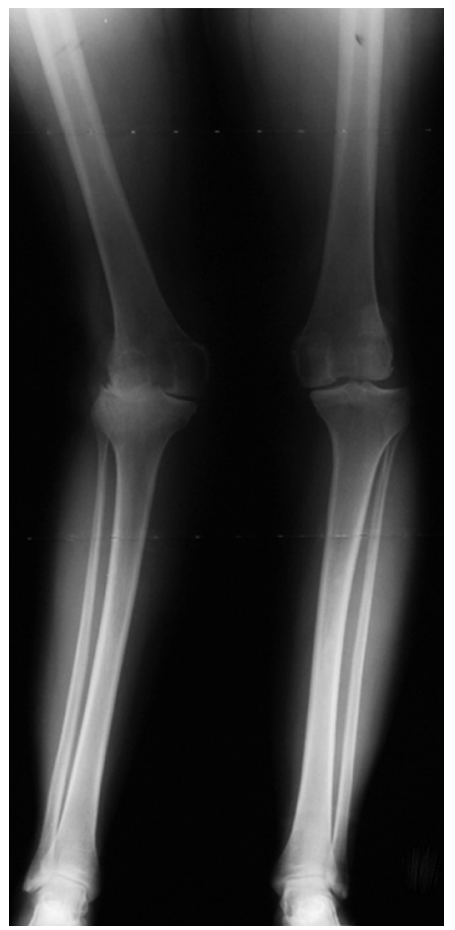

Fig. 1a

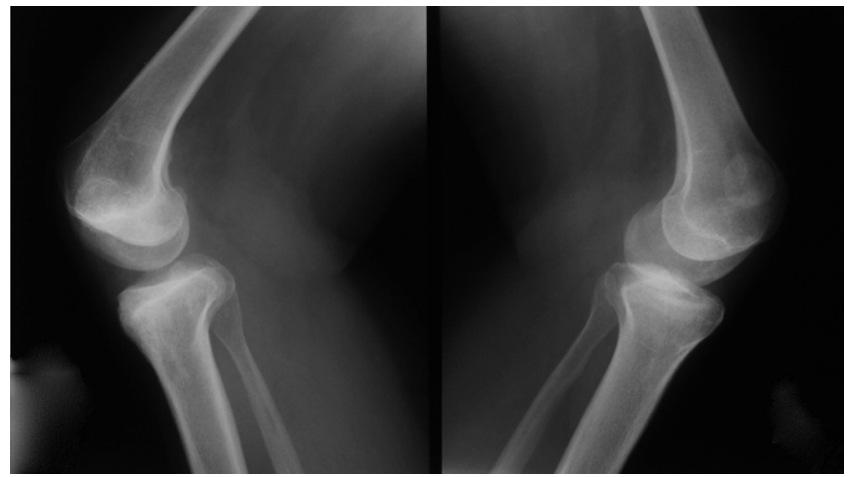

Fig. 1b

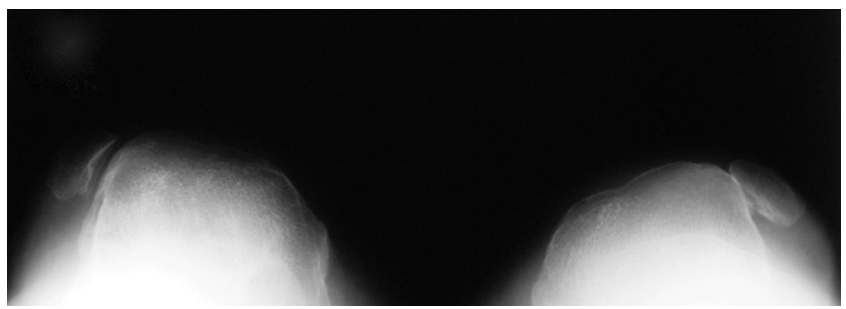

Fig. 1c

Pre-operative radiographs showing a) anteroposterior, b) lateral and c) skyline views in the patient with bilateral patellar dislocation.

in the collateral ligaments. ${ }^{8}$ However, when external tibial torsion is between $35^{\circ}$ and $45^{\circ}$ standard TKR can be reliably performed by using a posterior stabilised implant, with insertion of the tibial component in external rotation. ${ }^{8}$

We report our experience with simultaneous internal rotational osteotomy of the proximal tibia and TKR in patients with osteoarthritis and patellar instability secondary to external tibial torsion $>45^{\circ}$.

\section{Patients and Methods}

The series consisted of eight women (ten knees) with osteoarthritis and patellar instability secondary to severe external tibial torsion. One patient (both knees) had neglected irreducible patellae (Fig. 1) and seven (eight knees) had a history of recurrent dislocation of the patella. Their mean age was 59.4 years (54 to 64 ) at operation. All underwent simultaneous TKR and proximal internal tibial rotational osteotomy by the senior author (JCC). The mean follow-up was 47.2 months (24 to 120 ).

Six patients had various operations previously. Two had undergone the Hauser procedure, ${ }^{9}$ three had transfer of the patellar tendon by medialisation of the tibial tuberosity and one a lateral release with medial plication and patellar tendon transfer.

Pre-operative assessment included examination of the torsional alignment of the lower limb as discussed above, the stability of the knee and the range of movement and a distal neurovascular examination. In all patients, external tibial torsion was determined by measuring the thigh-foot angle in the prone position using a goniometer. ${ }^{7}$ The mean external tibial torsion was $57.5^{\circ}\left(50^{\circ}\right.$ to $\left.65^{\circ}\right)$. In the seven patients (eight knees) with recurrent patellar dislocations, the $\mathrm{Q}$ angle was measured pre- and post-operatively. It could not be measured clinically in the patient with dislocated patellae.

Radiological evaluation included standing anteroposterior (AP), lateral and skyline views with the knee flexed at $30^{\circ}$. Also, a three-foot standing radiograph with the feet pointing forwards was taken to determine the mechanical axis of the limbs. Any internal rotation of the femur shown in this view supported the diagnosis of torsional deformity. Since the external tibial torsion in these patients was so obvious, further imaging, such as CT, was not required. All the knee components were posterior stabilised (Nexgen, LPS; Zimmer, Warsaw, Indiana).

Clinical and functional evaluations were carried out by comparing the pre- and post-operative Knee Society scores $^{10}$ (200 points). This system includes a 100 -point knee score for the evaluation of categories such as pain, range of movement and stability, with deductions for flexion contracture, extension lag, instability and malalignment. The functional component has a maximum of 100 points for parameters such as the ability to walk on level ground and on stairs, as well as the necessity for walking aids. The post-operative scores were based on the most recent follow-up examination. 


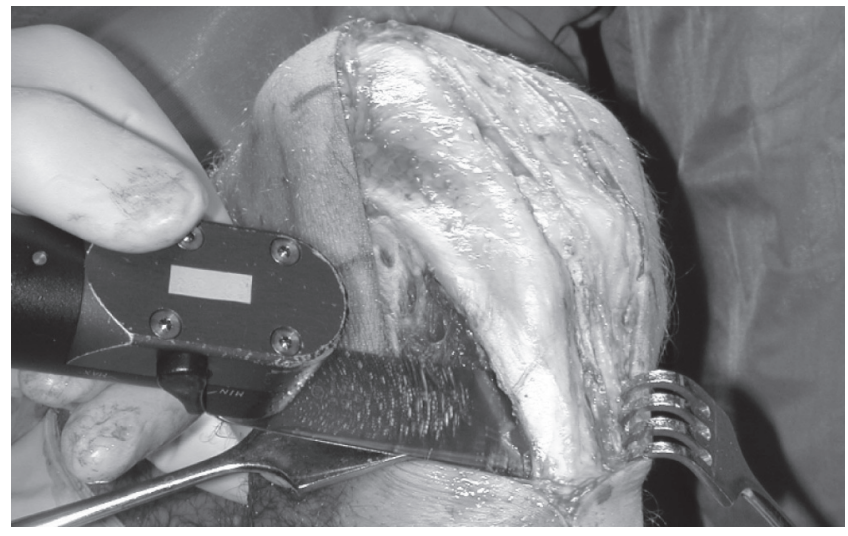

Fig. 2a

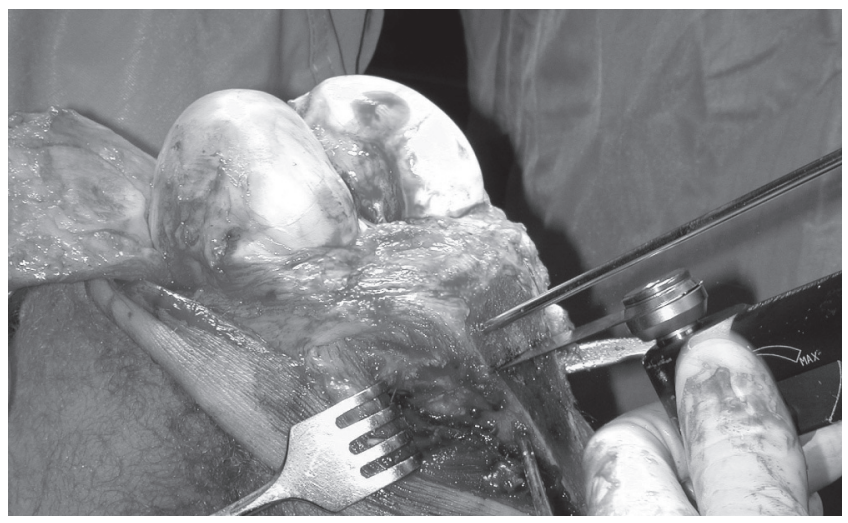

Fig. 2c

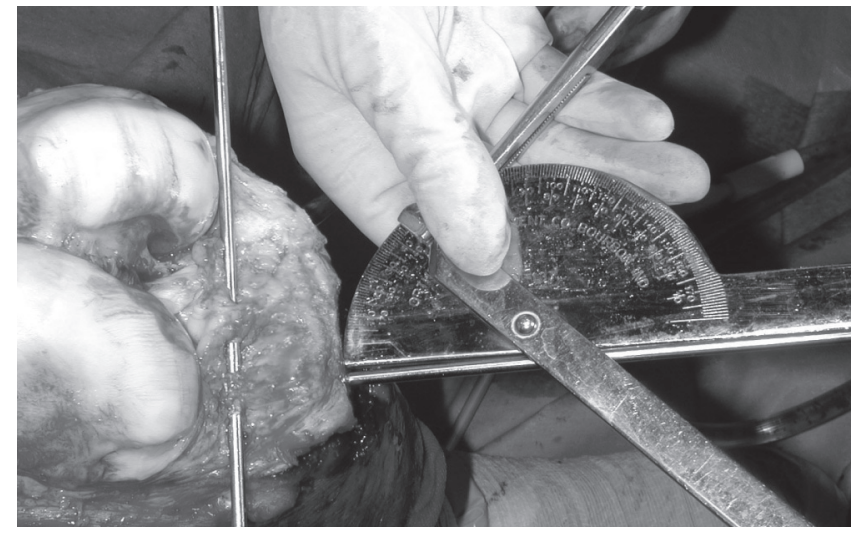

Fig. 2b

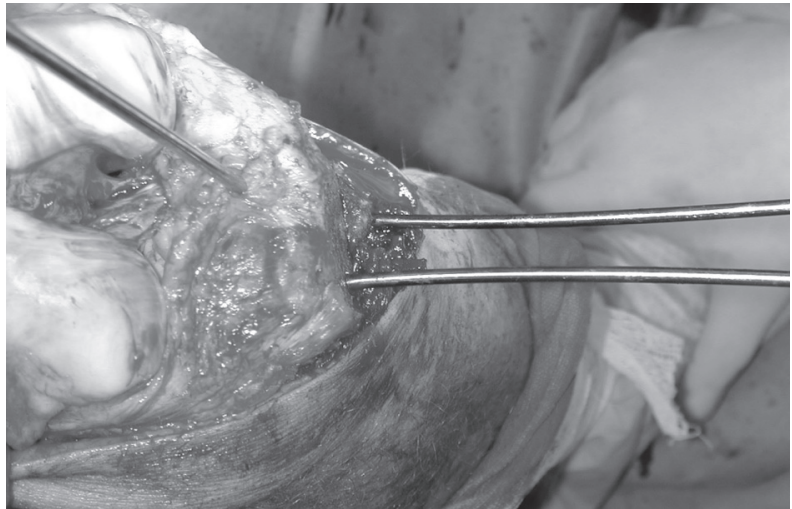

Fig. 2d

Photographs showing the stages of rotational proximal tibial osteotomy (a) Osteotomy of the tibial tuberosity. (b) A Kirschner (K)-wire is inserted intc the proximal tibia from anterior to posterior parallel to the joint line and a second K-wire inserted in external rotation relative to the degree of correction required. (c) The osteotomy is performed with an oscillating saw $4 \mathrm{~cm}$ to $4.5 \mathrm{~cm}$ from the joint line. (d) The ankle is internally rotated until the two wires are parallel.

All the patients were asked to answer a questionnaire which included their levels of satisfaction post-operatively, their willingness to have the operation done again (in unilateral cases) and the time to achieve full rehabilitation.

Operative technique. All operations were undertaken through a midline medial parapatellar approach. A coronal osteotomy of the tibial tuberosity $1.0 \mathrm{~cm}$ to $1.5 \mathrm{~cm}$ in thickness and extending $5 \mathrm{~cm}$ to $6 \mathrm{~cm}$ in length was performed to mobilise the patellar tendon (Fig. 2a). ${ }^{11}$ The tuberosity was reflected superiorly. The femur was prepared with standard cuts.

The proximal tibia was then resected in a standard fashion using an extramedullary guide. The tibia was reamed to a length of $145 \mathrm{~mm}$ to accommodate the stemmed prosthesis which would stabilise the rotational osteotomy, the level of which was marked $4.5 \mathrm{~cm}$ below the proximal tibial resection, thereby allowing a metaphyseal fit for the tibial component.

The inner aspect of the fibular head was resected, leaving its lateral cortex and attachment of the lateral collateral ligament intact to protect the peroneal nerve. Two Kirschner $(\mathrm{K}-)$ wires were placed offset from each other, based on the degree of correction required, one above and one below the proposed osteotomy (Fig. 2b). A transverse osteotomy was then performed (Fig. 2c) and the distal tibia internally rotated until the two offset K-wires were parallel (Fig. 2d).

The tibia was prepared and a stemmed tibial component inserted with cement. Care was taken to prevent extrusion of cement into the osteotomy. A buttress plate or staples were used if the rotational osteotomy was not fully stabilised by the stem (two patients). The tibial tuberosity was fixed by two AO cancellous screws and washers. The rest of the procedure was standard. The patients remained non-weightbearing for six to eight weeks until the tibial osteotomy had solidly united (Fig. 3).

Statistical analysis. The analysis of variance (ANOVA) test was used to compare pre- and post-operative knee scores. A p-value of $<0.05$ was chosen as being statistically significant.

\section{Results}

The mean pre-operative knee score improved significantly $(\mathrm{p}=0.0001)$ from 29.7 (15 to 39$)$ to 71.4 points (40 to 88 ). Similarly, the mean functional knee scores improved 

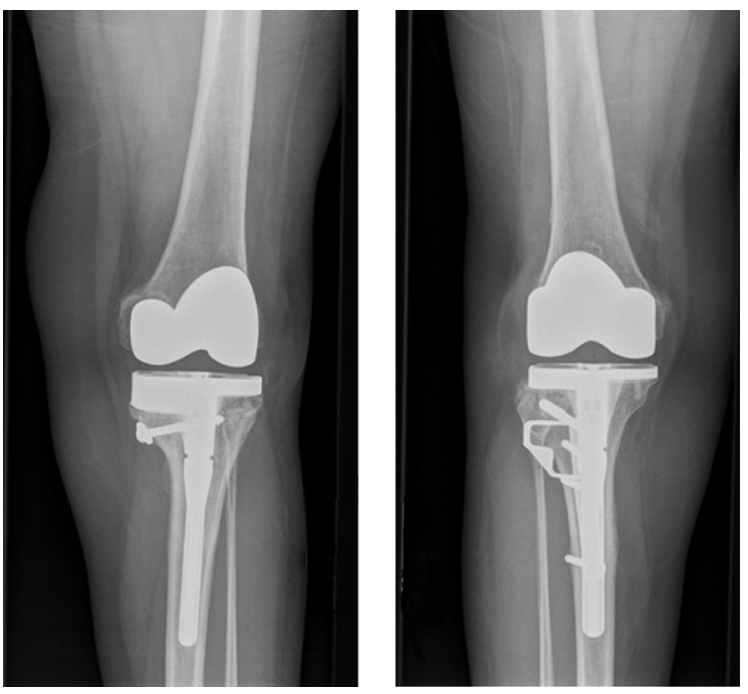

Fig. 3a
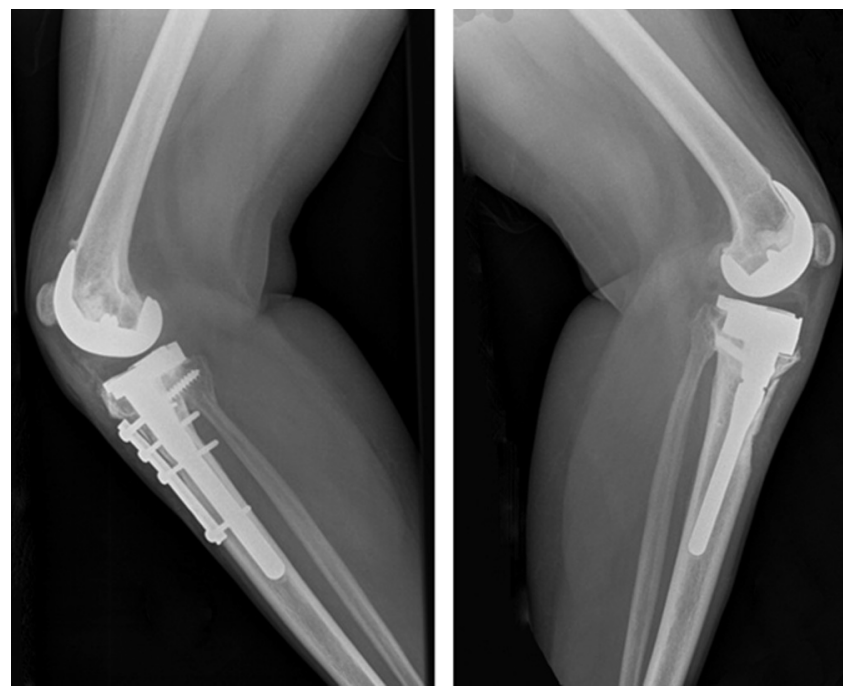

Fig. $3 b$
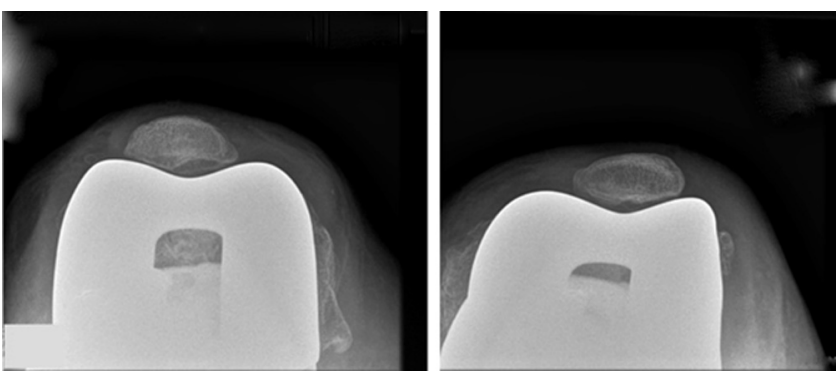

Fig. 3c

Post-operative radiographs showing a) AP, b) lateral and c) skyline views with good healing of the osteotomies (proximal tibia and tibial tubercle) in the patient with bilateral patellar dislocation.

significantly (ANOVA, $\mathrm{p}=0.0001)$ from $41.5(20$ to 65$)$ to 73.5 points $(60$ to 85$)$ at the most recent follow-up.

Pre-operative flexion contractures of $10^{\circ}$ or more were recorded in eight knees. Post-operatively, only one patient had a persistent flexion contracture of $10^{\circ}$ or more. The mean arc of flexion improved significantly (ANOVA $\mathrm{p}<0.0001)$ from $76.5^{\circ}\left(50^{\circ}\right.$ to $\left.90^{\circ}\right)$ pre-operatively to $104.5^{\circ}\left(65^{\circ}\right.$ to $\left.115^{\circ}\right)$ post-operatively. The mean $\mathrm{Q}$ angle improved significantly from $29.6^{\circ}\left(24^{\circ}\right.$ to $\left.35^{\circ}\right)$ to $17.1^{\circ}$ $\left(15^{\circ}\right.$ to $19^{\circ}, \mathrm{p}=0.002, t$-test $)$.

All the rotational and tibial tuberosity osteotomies had healed by eight weeks. The post-operative mechanical axis was restored to within $2^{\circ}$ of normality in each patient. There was neither radiological evidence nor symptoms suggestive of loosening of the components. Wound complications were not encountered.

Two patients complained of prominent metalwork and are scheduled for removal of the screws attaching the tibial tuberosity. One had a stiff knee and refused to have this treated. She had two previous knee operations in an attempt to correct patellofemoral instability and is not satisfied with the outcome. The remaining seven patients were satisfied. They noted that the operation improved their quality of life and would have this procedure again. However, all seven felt that rehabilitation to full function took from six to 12 months.

\section{Discussion}

A favourable outcome after TKR depends on restoration of the mechanical axis, correct orientation of the prosthesis and soft-tissue balancing. Numerous studies have discussed the influence of rotational alignment of the tibial and femoral components on patellar tracking. ${ }^{12-14}$ Berger et $\mathrm{al}^{12}$ analysed the rotational alignment of TKR components in asymptomatic patients with normal axial alignment $\left(1^{\circ}\right.$ of varus to $2^{\circ}$ of valgus) by CT and found that the combined amount of internal rotation of the femoral and tibial components correlated directly with the severity of patellofemoral instability. In patients who had patellofemoral complications, those with a combined internal rotation of $1^{\circ}$ to $4^{\circ}$ had lateral tracking and patellar tilting, those with $3^{\circ}$ to $8^{\circ}$ had patellar subluxation and those with $7^{\circ}$ to $17^{\circ}$ 
suffered from either early patellar dislocation before two years or failure of the patellar prosthesis at between two and six years post-operatively. The restoration of patellar tracking remains a challenge and requires meticulous surgical technique.

A well-recognised reason for malrotation after TKR is reliance on the posterior condylar axis in valgus knees, in which there is a high incidence of hypoplasia of the lateral femoral condyle. This may deceive the surgeon when making the femoral cuts and lead to internal rotation of the femoral component. ${ }^{15}$ Moreover, tibial rotation is not always easily determined, especially in the presence of torsional abnormalities. ${ }^{16}$ The use of the medial aspect of the tibial tuberosity as a landmark and repeated flexion and extension with trial components do not always provide the surgeon with a reliable guide for the rotational alignment of the tibial components. ${ }^{17,18}$ During pre-operative assessment, surgeons should be aware of excessive external tibial torsion as an additional cause of patellar maltracking, which can present as a spectrum ranging from patellar instability to chronic irreducible dislocation. The index of suspicion for this under-recognised aetiology should be high when there is patellar instability in valgus knees without lateral femoral condylar hypoplasia.

Previous studies for the treatment of chronic irreducible dislocation of the patella with arthritis have reported the use of augmented and stemmed TKR. ${ }^{19,20}$ These indicated that ligament balancing in such cases was difficult and they suggested the use of constrained components with patients often requiring extensive lateral release. The ensuing complications included wound breakdown and peroneal nerve palsy. ${ }^{19,20}$

Cameron ${ }^{8}$ found that external tibial torsion of up to $35^{\circ}$ to $45^{\circ}$ associated with osteoarthritis of the knee can be treated by intra-articular correction using a posterior stabilised TKR. This can be achieved by increasing the external rotation of the tibial component, which often requires downsizing to prevent anteromedial overhang. The correction which can be achieved reliably by this technique is limited, since beyond $20^{\circ}$ of intra-articular correction, there is a significant risk of increasing the tension in the collateral ligaments and exposing the central post to high torsional stresses. Therefore, when external tibial torsion is $>45^{\circ}$ a rotational osteotomy of the proximal tibia as described above should be considered. It can be performed either staged or simultaneously with TKR.

The senior author previously reported a good to excellent outcome in $14(77.8 \%)$ of a series of 18 patients with recurrent patellar dislocation and increased $\mathrm{Q}$ angle due to excessive external tibial torsion and patella alta in younger patients without arthritis. ${ }^{1}$ The keystone of the technique is a tibial derotational osteotomy which realigns the extensor mechanism and addresses the aetiology directly, without requiring an extensive soft-tissue release. ${ }^{1}$ Similar principles were applied concomitantly with TKR in our study and six of the eight patients had undergone previous tibial tuberosity realignment procedures for patellar instability. Medialisation of the extensor mechanism to a more anatomically correct alignment in patients with major external tibial torsion can be obtained only by derotation of the tibia because of the technical restrictions of current procedures for medialisation of the tibial tuberosity which have a maximal correction of $20^{\circ} .{ }^{21-23}$

We were able to restore the $\mathrm{Q}$ angle in all patients in our study by derotational osteotomy. There was no need for constrained implants and no wound complications occurred. All the osteotomies healed uneventfully and at a mean of four years, none of the knees required revision and there was no radiological or clinical evidence of loosening.

Patients should be informed that the post-operative rehabilitation is expected to take about a year. Also, irritation from hardware is a possible problem which can be addressed by its elective removal.

One limitation of our study was the relatively short follow-up. However, we believe that it was enough to uncover any problem related to the concomitant use of the osteotomy with TKR. The second limitation was the relatively small number of patients, but to the best of our knowledge this was the first series reporting this method of treating the rare combination of osteoarthritis and severe external tibial torsion.

We believe that, although demanding, the technique is effective in such patients.

No benefits in any form have been received or will be received from a commercial party related directly or indirectly to the subject of this article.

\section{References}

1. Cameron JC, Saha S. External tibial torsion: an under recognized cause of recurrent patellar dislocation. Clin Orthop 1996;328:177-84

2. Colvin AC, West RV. Review article: patellar instability. J Bone Joint Surg [Am] 2008;90-A:2751-62.

3. Turner MS, Smillie IS. The effect of tibial torsion on the pathology of the knee. $J$ Bone Joint Surg [Br] 1981;63-B:396-8.

4. Eckhoff DG. Effect of limb malrotation on malalignment and osteoarthritis. Orthop Clin North Am 1994;25:405-14.

5. Guidera KJ, Ganey TM, Keneally CR, Ogden JA. The embryology of lower extremity torsion. Clin Orthop 1994;302:17-21.

6. Beaconsfield T, Pintore E, Maffulli N, Petrie GJ. Radiological measurements in patellofemoral disorders. Clin Orthop 1994;308:18-28.

7. Stuberg W, Temme J, Kaplan P, Clarke A, Fuchs R. Measurement of tibial torsion and thigh-foot angle using goniometry and computed tomography. Clin Orthop 1991;272:208-12.

8. Cameron HU. A comedy of errors: the bad knee. J Arthroplasty 2005;4(Suppl 2):1822.

9. Chrisman OD, Snook GA, Wilson TC. A long-term prospective study of the Hauser and Roux-Goldthwait procedures for recurrent patellar dislocation. Clin Orthop 1979;144:27-30.

10. Insall JN, Dorr LD, Scott RD, Scott WN. Rationale of the Knee Society clinical rating system. Clin Orthop 1989;248:13-14.

11. AI-Sayyad MJ, Cameron JC. Functional outcome after tibial tubercle transfer for the painful patella alta. Clin Orthop 2002;396:152-62.

12. Berger RA, Crossett LS, Jacobs JJ, Rubash HE. Malrotation causing patellofemoral complications after total knee arthroplasty. Clin Orthop 1998;356:144-53.

13. Barrack RL, Schrader T, Bertot AJ, Wolfe MW, Myers L. Component rotation and anterior knee pain after total knee arthroplasty. Clin Orthop 2001;392:46-55.

14. Eckhoff DG, Piatt BE, Gnadinger CA, Blaschke RC. Assessing rotational alignment in total knee arthroplasty. Clin Orthop 1995;318:176-81.

15. Pagnano MW, Hanssen AD. Varus tibial joint line obliquity: a potential cause of femoral component malrotation. Clin Orthop 2001;392:68-74. 
16. Nagamine R, Miyanishi K, Miura H, et al. Medial torsion of the tibia in Japanese patients with osteoarthritis of the knee. Clin Orthop 2003;408:218-24.

17. Uehara K, Kadoya Y, Kobayashi A, Ohashi H, Yamano Y. Bone anatomy and rotational alignment in total knee arthroplasty. Clin Orthop 2002;402:196-201.

18. Ikeuchi M, Yamanaka N, Okanoue Y, Ueta E, Tani T. Determining the rotational alignment of the tibial component at total knee replacement: a comparison of two techniques. J Bone Joint Surg [Br] 2007;89-B:45-9.

19. Stern SH, Moeckel BH, Insall Jl. Total knee arthroplasty in valgus knees. Clin Orthop 1991;273:5-8
20. Rose HA, Hood RW, Otis JC, Ranawat CS, Insall JI. Peroneal nerve palsy following total knee arthroplasty. J Bone Joint Surg [Am] 1982;64-A:347-51.

21. Fulkerson JP, Becker GJ, Meaney JA, Miranda M, Folcik MA. Anteromedial tubercle transfer without bonegraft. Am J Sports Med 1990;18:490-7.

22. Miller BJ, Larochelle PJ. The treatment of patellofemoral pain by combined rotation and elevation of the tibial tubercle. J Bone Joint Surg [Am] 1986;68-A:419-23.

23. Noll BJ, Ben-Itzhak I, Rossouw P. Modified tibial tubercle elevation with realignment for patellofemoral pain. Clin Orthop 1988;234:178-82. 